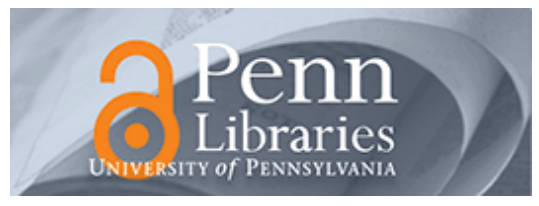

University of Pennsylvania

ScholarlyCommons

2-2009

\title{
Diabetes Causes the Accelerated Loss of Cartilage During Fracture Repair Which Is Reversed by Insulin Treatment
}

\author{
Rayyan A. Kayal \\ Jazia Alblowi \\ Erin McKenzie \\ Nanarao Krothapalli \\ Lee Silkman
}

See next page for additional authors

Follow this and additional works at: https://repository.upenn.edu/dental_papers

Part of the Dentistry Commons, Endocrinology, Diabetes, and Metabolism Commons, and the Osteopathic Medicine and Osteopathy Commons

\section{Recommended Citation}

Kayal, R. A., Alblowi, J., McKenzie, E., Krothapalli, N., Silkman, L., Gerstenfeld, L., Einhorn, T., \& Graves, D. T. (2009). Diabetes Causes the Accelerated Loss of Cartilage During Fracture Repair Which Is Reversed by Insulin Treatment. Bone, 44 (2), 357-363. http://dx.doi.org/10.1016/j.bone.2008.10.042

At the time of publication, author Dana Graves was affiliated with Boston University School of Dental Medicine. Currently, he is a faculty member at the Penn Dental School at the University of Pennsylvania.

This paper is posted at ScholarlyCommons. https://repository.upenn.edu/dental_papers/7

For more information, please contact repository@pobox.upenn.edu. 


\title{
Diabetes Causes the Accelerated Loss of Cartilage During Fracture Repair Which Is Reversed by Insulin Treatment
}

\begin{abstract}
Fracture healing in diabetic individuals and in animal models of diabetes is impaired. To investigate mechanisms by which diabetes may affect fracture healing we focused on the transition from cartilage to bone, a midpoint in the fracture healing process. Femoral fractures were induced in mice rendered diabetic by multiple low dose streptozotocin treatment and compared to matching normoglycemic mice. One group of diabetic animals was treated with slow release insulin to maintain normal serum glucose levels. The results indicate that there was relatively little difference in the initial formation of the fracture callus on day 10 . However, on day 16 the diabetic group had significantly smaller callus, greater loss of cartilage and enhanced osteoclastogenesis that was normalized by treatment with insulin when assessed by histomorphometric analysis. Chondrocyte apoptosis was significantly higher in diabetic mice and this increase was blocked by insulin. These changes were accompanied by diabetes-increased mRNA levels of RANKL, TNF- $\alpha$, and ADAMTS- 4 and -5 measured by real-time PCR, which was reversed by insulin treatment. On days 16 and 22 bone formation within the callus of diabetic mice was significantly less than the normoglycemic and brought to normal levels by insulin treatment. These results suggest that a significant effect of diabetes on fracture healing is increased chondrocyte apoptosis and osteoclastogenesis that accelerates the loss of cartilage and reduces the anlage for endochondral bone formation during fracture repair. That insulin reverses these effects demonstrates that they are directly related to the diabetic condition.
\end{abstract}

\section{Keywords}

Fracture, Femur, Diabetes, Hyperglycemia, Healing, Insulin, Cartilage, Apoptosis

\section{Disciplines}

Dentistry | Endocrinology, Diabetes, and Metabolism | Osteopathic Medicine and Osteopathy

\section{Comments}

At the time of publication, author Dana Graves was affiliated with Boston University School of Dental Medicine. Currently, he is a faculty member at the Penn Dental School at the University of Pennsylvania.

\section{Author(s)}

Rayyan A. Kayal, Jazia Alblowi, Erin McKenzie, Nanarao Krothapalli, Lee Silkman, Louis Gerstenfeld, Thomas Einhorn, and Dana T. Graves 


\title{
Diabetes causes the accelerated loss of cartilage during fracture repair which is reversed by insulin treatment
}

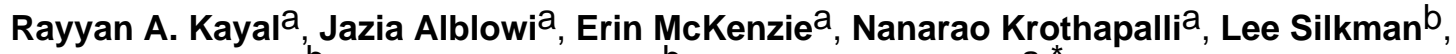 \\ Louis Gerstenfeld ${ }^{b}$, Thomas A. Einhorn ${ }^{b}$, and Dana T. Graves ${ }^{a}{ }^{*}$ \\ aDepartment of Periodontology and Oral Biology, Boston University School of Dental Medicine, Boston, MA \\ 02118, USA \\ bDepartment of Orthopedic Surgery, Boston University School of Medicine, Boston, MA 02118, USA
}

\section{Abstract}

Fracture healing in diabetic individuals and in animal models of diabetes is impaired. To investigate mechanisms by which diabetes may affect fracture healing we focused on the transition from cartilage to bone, a midpoint in the fracture healing process. Femoral fractures were induced in mice rendered diabetic by multiple low dose streptozotocin treatment and compared to matching normoglycemic mice. One group of diabetic animals was treated with slow release insulin to maintain normal serum glucose levels. The results indicate that there was relatively little difference in the initial formation of the fracture callus on day 10 . However, on day 16 the diabetic group had significantly smaller callus, greater loss of cartilage and enhanced osteoclastogenesis that was normalized by treatment with insulin when assessed by histomorphometric analysis. Chondrocyte apoptosis was significantly higher in diabetic mice and this increase was blocked by insulin. These changes were accompanied by diabetes-increased mRNA levels of RANKL, TNF- $\alpha$, and ADAMTS-4 and -5 measured by realtime PCR, which was reversed by insulin treatment. On days 16 and 22 bone formation within the callus of diabetic mice was significantly less than the normoglycemic and brought to normal levels by insulin treatment. These results suggest that a significant effect of diabetes on fracture healing is increased chondrocyte apoptosis and osteoclastogenesis that accelerates the loss of cartilage and reduces the anlage for endochondral bone formation during fracture repair. That insulin reverses these effects demonstrates that they are directly related to the diabetic condition.

\section{Keywords}

Fracture; Femur; Diabetes; Hyperglycemia; Healing; Insulin; Cartilage; Apoptosis

\section{Introduction}

Fracture healing is a complicated multi-phase process that involves the coordinated activity of many cell types [1]. The healing process is initiated by a hematoma that forms in response to the disruption of blood vessels. Progenitors are then recruited to the site of injury where they proliferate and differentiate into chondrocytes and osteoblasts. Chondrocytes produce cartilage forming a soft cartilaginous callus, which calcifies and protects the injured site. As chondrocytes undergo apoptosis osteoclasts begin the removal of mineralized cartilage, setting

(C) 2008 Published by Elsevier Inc.

*Corresponding author. E-mail address: E-mail: rdgraves@bu.edu (D.T. Graves).

Edited by: D. Burr 
the stage for endochondral bone formation by osteoblasts. The bony callus then undergoes remodeling until the bone reaches it original form.

Bone is affected by diabetes, which causes osteopenia and impairs fracture healing [2,3]. Osteopenia is thought to be a contributing factor to the increased fracture risk observed in diabetic patients [4-6]. Most studies on osteopenia have focused on impaired bone formation, which is supported by a decrease in bone mineral density and reduced markers of bone formation such as serum levels of osteocalcin and alkaline phosphatase [7-9]. Several mechanisms have been suggested including changes in cell signaling caused by hyperglycemia, inflammation associated with diabetes, changes in circulating growth factors and endocrine hormones, greater oxidative stress and increased cell death $[2,8,10]$. Krakauer et al. have suggested that patients with diabetes have reduced bone formation and bone accumulation during growth, while later in life hyperglycemia leads to increased bone resorption and osteopenia [11]. Recent evidence supports the concept that diabetes can contribute to osteopenia by increasing osteoclast formation [11-14].

Case reports and clinical investigations have reported that diabetes delays union of healing fractures and increases healing time in diabetic subjects compared to matched controls $[5,15$, 16]. Animal models also demonstrate that diabetes leads to the formation of smaller calluses with decreased bone and cartilage formation, decreased proliferation and differentiation of osteoblastic cells and chondrocytes and a two-fold reduction in the mechanical strength during fracture repair in diabetic compared to normoglycemic animals [17-21]. DNA content is decreased by $40 \%$ in healing diabetic fractures compared to controls, an indication that the diabetic calluses have decreased cellularity [22]. This could be due a decrease in the rate of cell proliferation associated with decreased growth factor production [23]. In addition, there is a decrease in the collagen content of the callus of the diabetic animals compared with normoglycemic animals $[19,22]$. A decrease in matrix could results from reduced formation of osteoblasts that produce bone [24].

We previously investigated the impact of diabetes on fracture healing in the tibia. The results identified a previously unrecognized catabolic effect of diabetes on fracture repair, the accelerated loss of cartilage in the diabetic group [25]. To investigate further the impact of diabetes on endochondral bone formation experiments were carried out where fractures were induced in the femur and the impact of diabeteswas tested by treating mice with slow release insulin. Histologic and molecular analysis indicated that diabetes caused an increased osteoclastogenesis and loss of cartilage and increased mRNA levels of several pro-resorptive factors. Each of these parameters was reversed by treatment with insulin. These studies represent an important extension of our previous results since they demonstrate that the catabolic events are specifically related to the diabetic state since they are rescued by insulin treatment.

\section{Materials and methods}

\section{Induction of type 1 diabetes}

All experiments were approved by the Boston University Medical Center Institutional Animal Care and Use Committee (IACUC). Eight week old, male CD-1 mice purchased from Charles River Laboratories (Wilmington, MA) were rendered diabetic by intraperitoneal injections (i.p.) of streptozotocin $(40 \mathrm{mg} / \mathrm{kg}$ ) (Sigma, St. Louis,MO) in $10 \mathrm{mM}$ citrate buffer daily for 5 days [26]. Control mice were treated identically with vehicle alone, $10 \mathrm{mM}$ citrate buffer. A group of diabetic mice received insulin treatment through slow release insulin implants (Linbit, Linshin Canada, Toronto, ON) placed subcutaneously. These implants release $\sim 0.1 \mathrm{U}$ of insulin per day and depending on the weight of the animal, each animal received 4 to 5 implant according to the manufacturer's instructions. Animals were considered to be diabetic when 
serum glucose levels exceeded $250 \mathrm{mg} / \mathrm{dl}$ (Accu-Chek, Roche Diagnostics, Indianapolis, IN) and were measured weekly. Glycosylated hemoglobin levels were measured at the time of euthanasia by Glyco-tek affinity chromatography (Helena Laboratories, Beaumont, TX) (Table 1).

\section{Femoral fractures}

All studies were performed on male mice that were diabetic for 3 weeks prior to fracture. A simple transverse closed fracture of the femur was performed in separate animals as previously described $[1,25,27]$. In the femoral fractures, the incision was made lateral to the knee to expose the articular surface of the femur. Access to the medullary canal was gained with a 25 gauge needle and a 27 gauge spinal needle was inserted for fixation. After closure of the incision, a fracture was created by blunt trauma. The status of the fracture was confirmed at the time of euthanasia radiographically and any fractures not consistent with standardized placement criteria (mid-diaphyseal) or grossly comminuted were excluded. Animals were subsequently euthanized at 10, 16 and 22 days. Femurs were harvested and most of the associated muscle and soft connective tissue was gently removed.

\section{Histomorphometric analysis}

The femurs with a small amount of surrounding muscle and soft tissues were fixed for $72 \mathrm{~h}$ in cold 4\% paraformaldehyde and decalcified for 2 weeks by incubation in cold Immunocal (Decal Corporation, Congers, NY). After decalcification, specimens were embedded in paraffin and the fractures were cut in transverse cross-sections at $5 \mu \mathrm{m}$ and prepared for staining. To obtain mean values for a given specimen three different histologic sections were examined that included a histologic section at the fracture line and histologic sections $0.5 \mathrm{~mm}$ proximal and medial to this section. These three sections represent the central region of the fracture callus. The callus, cartilage and newbone area were measured in sections stained with hematoxylin and eosin $(\mathrm{H} \& \mathrm{E})$, safranin-O/fast green, and Masson trichrome respectively. The area of each tissue was assessed by computer assisted image analysis using Image ProPlus software (Media Cybernetics, Silver Spring, MD) as previously described [28]. Osteoclast formation was determined by counting the number of multinucleated, tartrate resistant acid phosphatase (TRAP) positive cells lining bone or cartilage as previously described [28]. Apoptotic cells were detected by the TUNEL assay (ApopTag® Peroxidase In Situ Apoptosis Detection Kit, Chemicon International, Temecula, CA). For cartilage area and osteoclast counts measurements across the callus are presented $(0,0.5 \mathrm{~mm}$ proximal and medial and $1.0 \mathrm{~mm}$ proximal and medial of the fracture line) in addition to mean values per specimen. For histologic analysis the following number of animals were examined per group with each animal as the unit of measurement, 10 day (normal, $n=8$, diabetic, $n=9$, diabetic insulin treated, $n=7$ ) and for 16 and 22 day specimens $n=6$ for all groups. Measurements were made by one examiner under blinded conditions, with the results confirmed by a second examiner.

\section{RNA isolation and qPCR}

After euthanasia, fracture calluses were carefully dissected removing all muscle and non-callus tissue and immediately frozen in liquid nitrogen. Total RNA was extracted with Trizol (Life Technologies, Rockville, MD) from pulverized frozen tissue and further purified by an RNAeasy MinElute cleanup kit (Qiagen, Valencia, CA). The concentration and integrity of the extracted RNA was verified by $260 \mathrm{~nm} / 280 \mathrm{~nm}$ spectro-photometry and denaturing agarose gel electrophoresis with ethidium bromide staining. For a given experiment RNA from 3 specimens was combined and TaqMan reagents were used for first-strand cDNA synthesis and amplification (Applied Biosystems, Foster City, CA) and three sets of experiments were performed involving a total of 9 mice per data point. Quantitative real time PCR was used to assess mRNA levels of each genes of interest related to inflammation, osteoclastogenesis, 
matrix degradation and cartilage formation (TNF- $\alpha$, RANKL, RANK, OPG, ADAMTS-4 and -5 , and collagen-II and $-\mathrm{X}$ ). Specific primers and probe sets were obtained from Applied Biosystems. Results were normalized with an $18 \mathrm{~S}$ ribosomal primer and probe set. Each experiment was performed three times and the results from the three separate experiments were combined in order to derive mean values. For a given gene, the expression for each group was set relative to the value obtained for the normoglycemic control animals on day 10. For statistical purposes $n=3$.

\section{Statistical analysis}

Data are presented as mean values \pm SEM. Two-way ANOVA was used to test whither the group (normal, diabetic and insulin treated) and/or time point had a significant effect. It was also used to find statistical interactions between group and time. Interaction was discovered in some qPCR results (TNF- $\alpha$, RANKL, TRAP). The Bonferonni method of correction was applied to this data and the differences were shown to be still significant. One-way ANOVA with Scheffe's post-hoc test was used to analyze differences between multiple groups at a give time point andwas also used to analyze differences between time points in the same group.

\section{Results}

\section{Callus and cartilage area}

To focus on the transition from cartilage to bone specimens were collected at 10, 16 and 22 days after the fracture. Callus size was assessed at the fracture line and $0.5 \mathrm{~mm}$ on the proximal and distal (Fig. 1A). Results from these measurements indicated that the callus size was not significantly different in the diabetic compared to normoglycemic groups on day 10 , but just missed statistical significance $(P=0.07)$. On day 16 the normoglycemic group had 1.8 fold larger callus area per section than the diabetic and the insulin treated group had 1.7 fold larger callus area than the untreated diabetic animals $(P<0.05)$. The smaller callus area in the diabetic group was maintained on day $22(P<0.05)$.

The impact of diabetes and insulin treatment on cartilage was examined. Ten days after fracture the normoglycemic and insulin treated diabetic animals had 1.6 fold more cartilage area than the diabetic $(P<0.05$; Fig. 1B). However, no statistical difference was found when collagen-II and -X mRNA levels were measured (Fig. 1C). A striking difference was noted over time. From day 10 to 16 the cartilage area in the diabetic group decreased by $42 \%(P<0.05)$ while the normoglycemic and diabetic insulin treated groups showed no change. On day 16 the normoglycemic animals had fractures with 2.4 fold more cartilage than the diabetic group and insulin treatment reversed this deficit $(P<0.05)$. From day 16 to day 22 there was a significant reduction in cartilage in all three groups $(P<0.05)$. On day 22 the amount of total cartilage in all groups was small and similar $(P>0.05)$. Changes in cartilage were also examined at $0.5 \mathrm{~mm}$ intervals along the $2 \mathrm{~mm}$ length of the callus (Fig. 2). On both days 10 and 16 the area of cartilage was larger in the normoglycemic group at all sections compared to the diabetic $(P<0.05$; Figs. 2A and B). The insulin treated group was also larger than the diabetic group $(P<0.05)$ and equivalent to the normal.

\section{Osteoclast numbers}

On day 10 osteoclast numbers were low in all groups but significantly higher in the diabetic than the normoglycemic or insulin treated diabetic mice $(P<0.05$; Fig. $3 \mathrm{~A})$. On day 16 there were 1.75 fold more osteoclasts in the diabetic group compared to the normoglycemic group $(P<0.05)$, which was reversed by insulin treatment $(P<0.05)$. On day 22 the normoglycemic, diabetic and diabetic insulin treated groups showed a similar number of osteoclasts. Day 16 specimens were examined in more detail to assess the distribution of osteoclasts over a wider range of the fracture callus (Fig. 3B). The increase in osteoclast numbers in the diabetic group 
was generally higher across the fracture callus compared to the normoglycemic mice and insulin treatment reversed this increase across the callus.

\section{mRNA levels of pro-resorptive factors}

To gain insight into mechanism that may causes diabetes enhanced osteoclastogenesis mRNA levels TNF- $\alpha$, RANKL, RANK, and OPG were measured (Fig. 4). On day 16, TNF- $\alpha$ mRNA levels were almost 2 fold higher in diabetic fracture calluses compared to the normoglycemic controls (Fig. 4A; $P>0.05$ ). The increase caused by diabetes was reduced to normal levels by treatment with insulin. On day $22 \mathrm{TNF}-\alpha$ was still significantly higher in the diabetic compared to the other two groups $(P<0.05)$. RANKL levels were low on day 10 and increased significantly by day 16 in the diabetic fracture calluses (Fig. 4B; $P<0.05$ ). On day 16 RANKL mRNA levels were almost 2 fold higher in the healing fractures of mice with diabetes versus normal control mice $(P<0.05)$. Slow release insulin reduced RANKL to normal levels $(P>0.05)$. In contrast to RANKL, them RNA levels of OPG and RANK did not show any difference between the groups (Figs. $4 \mathrm{C}$ and $\mathrm{D} ; P>0.05$ ).

The mRNA levels of ADAMTS-4 and -5 were examined in each group because of their role in degrading proteoglycan found in cartilage. On day 10 ADAMTS-4was similar in all three groups, but was 1.5 fold higher in the diabetic compared to the other two on day $16(P<0.05)$. On day 22 , when cartilage was largely gone in the fracture calluses of all groups, the levels were substantially less (Fig. 5A). The ADAMTS-5 levels were initially similar in all groups on day 10 but were significantly higher in diabetic mice on 16 compared to the matched normal control or insulin treated animals $(P<0.05$; Fig. 5B). On day 22 the levels were similar in all three groups $(P>0.05)$.

\section{Apoptosis}

To gain insight into how diabetes might affect other aspects of cartilage during endochondral bone formation we quantified the number of apoptotic chondrocytes and compared that to the total number of apoptotic cells per callus excluding hematopoietic tissue found on day 22 specimens (Fig. 6). The number of apoptotic chondrocytes was more than 5 -fold higher in the diabetic group compared to the normoglycemic $(P>0.05$; Fig. 6A). Insulin treatment reduced by number of apoptotic chondrocytes by $60 \%$, which approached but did not reach the level found in normoglycemic fracture calluses. When the whole callus was examined the diabetic group showed a 2.5 fold increase in apoptotic cells compared to normoglycemics, which was significantly reversed with insulin treatment $(P<0.05$; Fig. 6B).

\section{Bone formation}

The amount of new bone was small and equivalent in all groups on day $10(P>0.05$; Fig. 7). There was a significant increase in bone formation from day 10 to day 16 in the normoglycemic and diabetic insulin treated groups $(P<0.05)$ but not the diabetic untreated group. On day 16, the normoglycemic facture calluses had 1.8 fold more bone than the diabetic group $(P<0.05)$. With insulin treatment, the amount of new bone was restored to match the normoglycemic group. The difference in amount of bone between the different groups was maintained on day $22(P<0.05)$.

\section{Discussion}

In patients with diabetes there is an increase risk of fracture [3-6] and impairment in the fracture healing process [15,16]. Similar findings have been reported in animal models $[19,20,22,29]$. Diabetic animals exhibit fracture calluses that are smaller in size than those in normal animals $[19,21,23]$. We found that at an early time point, day 10, the difference between the diabetic and control groups just missed statistical significance. While the cartilage area was smaller in 
the diabetic group at this time point the expression of collagen-II and X was similar. This apparent discrepancy may be due to the impact of greater osteoclastogenesis in the diabetic group on day 10. A striking observation was the greater reduction in callus area between days 10 and 16 in the diabetic group associated with accelerated loss of cartilage. In contrast the normoglycemic and insulin treated diabetic mice did not exhibit a similar decrease. These findings represent an important extension of our previous work because the reversal with insulin treatment specifically links it to the diabetic condition. Moreover, diabetes resulted in a two-fold increase in osteoclasts on 16 and a two-fold increase in RANKL and TNF- $\alpha$. TNF$\alpha$ and RANKL are associated with the resorption of mineralized cartilage [30,31]. When osteoclast number is decreased during endochondral ossification, cartilage is not efficiently removed [32]. Furthermore, reduced TNF- $\alpha$ signaling or treatment with antibody that inhibits RANKL causes an increased retention of cartilage [33]. In the diabetic animals the opposite is observed, increased TNF- $\alpha$ and RANKL expression and the rapid and efficient removal of cartilage. Moreover, diabetes-increased TNF- $\alpha$ and RANKL was reversed by treatment with slow release insulin.

The mRNA levels of ADAMTS- 4 and -5 were assessed. ADAMTS-4 and -5 play an important role in degrading the proteoglycan matrix component of cartilage [34-37]. That ADAMTS-4 and -5 are higher in the diabetic group at day 16 would explain the greater removal of proteoglycan in the diabetic group and the fact that theywere reduced by insulin treatment indicates that their increase was an outcome of the diabetic condition. Diabetes also increased the number of apoptotic cells, especially chondrocytes, on day 16 . When diabetic animalswere treated with insulin, the number of apoptotic cells fell to normal levels. The loss of chondrocytes is thought to be physiologically important since chondrocyte cell death may generate signals that stimulate resorption of cartilage [38,39].

Diabetic fracture calluses were initially similar in size to the other two groups but became significantly smaller on days 16 and 22. Insulin treatment reversed this deficit. It is possible that the loss of cartilage, which serves as an anlagen for endochondral bone formation, contributes to the reduced bone formation and the reduced amount of bone contributes to the smaller callus size. The diminished callus size and reduced amount of bone may reduce the strength of the healing fracture callus, which has been reported by several investigators [1720].

We previously reported that the amount of cartilage that formed in tibial calluses were smaller in the diabetic but that this difference was not statistically significant. In the report here we found that the initial cartilage formation was statistically significant. It may also be due to slight differences in time points. However, there was consistency in several catabolic events that occur during fracture healing that were significantly increased by diabetes and which were reversed by insulin treatment. Other studies have shown that insulin treatment normalized the cellular proliferation, chondrogenesis, mineralization and mechanical strength of diabetic fracture healing $[17,40]$. The results suggest that many of the defects in fracture healing caused by diabetes are the direct result of hyperglycemia. However, it is also possible that hypoinsulinemia also contributes to these catabolic events. For example, insulin is a prosurvival factor for chondrocytes [29]. By stimulating survival of chondrocytes through insulin treatment the accelerated loss of cartilage may be affected. Furthermore, insulin has an anabolic effect on cartilage and bone [29] although the requirement of insulin during bone formation per se has been question by studies in which genetically modified euglyemic mice that lack insulin receptors in bone tissue do not exhibit altered bone formation or resorption [41]. Regardless of whether the primary effect of insulin is to reverse hyperglycemia or whether there are direct effects of insulin on cells, results presented here demonstrate the necessity of managing serum glucose levels during fracture healing as pointed out by other investigators $[17,20,42,43]$. 


\section{Acknowledgments}

This work was supported by a grant from National Institute of Arthritis and Musculoskeletal and Skin Diseases, PO1AR049920.

\section{References}

1. Gerstenfeld LC, Wronski TJ, Hollinger JO, Einhorn TA. Application of histomorphometric methods to the study of bone repair. J Bone Miner Res 2005;20:1715-1722. [PubMed: 16160729]

2. Jehle PM, Jehle DR, Mohan S, Bohm BO. Serum levels of insulin-like growth factor system components and relationship to bone metabolism in Type 1 and Type 2 diabetes mellitus patients. $\mathbf{J}$ Endocrinol 1998;159:297-306. [PubMed: 9795371]

3. Tuominen JT, Impivaara O, Puukka P, Ronnemaa T. Bone mineral density in patients with type 1 and type 2 diabetes. Diabetes Care 1999;22:1196-1200. [PubMed: 10388989]

4. Ivers RQ, Cumming RG, Mitchell P, Peduto AJ. Diabetes and risk of fracture: The Blue Mountains Eye Study. Diabetes Care 2001;24:1198-1203. [PubMed: 11423502]

5. Nicodemus KK, Folsom AR. Type 1 and type 2 diabetes and incident hip fractures in postmenopausal women. Diabetes Care 2001;24:1192-1197. [PubMed: 11423501]

6. Vestergaard P, Rejnmark L, Mosekilde L. Relative fracture risk in patients with diabetes mellitus, and the impact of insulin and oral antidiabetic medication on relative fracture risk. Diabetologia 2005;48:1292-1299. [PubMed: 15909154]

7. Botolin S, McCabe LR. Bone loss and increased bone adiposity in spontaneous and pharmacologically induced diabetic mice. Endocrinology 2007;148:198-205. [PubMed: 17053023]

8. Kemink SA, Hermus AR, Swinkels LM, Lutterman JA, Smals AG. Osteopenia in insulin-dependent diabetes mellitus; prevalence and aspects of pathophysiology. J Endocrinol Invest 2000;23:295-303. [PubMed: 10882147]

9. Hofbauer LC, Brueck CC, Singh SK, Dobnig H. Osteoporosis in patients with diabetes mellitus. J Bone Miner Res 2007;22:1317-1328. [PubMed: 17501667]

10. McCabe LR. Understanding the pathology and mechanisms of type I diabetic bone loss. J Cell Biochem 2007;102:1343-1357. [PubMed: 17975793]

11. Krakauer J, McKenna M, Burderer N, Rao D, Whitehouse F, Pafitt A. Bone loss and bone turnover in diabetes. Diabetes 1995;44:775-782. [PubMed: 7789645]

12. Suzuki K, Kurose T, Takizawa M, et al. Osteoclastic function is accelerated in male patients with type 2 diabetes mellitus: the preventive role of osteoclastogenesis inhibitory factor/osteoprotegerin (OCIF/OPG) on the decrease of bone mineral density. Diabetes Res Clin Pract 2005;68:117-125. [PubMed: 15860239]

13. Gough A, Abraha H, Li F, et al. Measurement of markers of osteoclast and osteoblast activity in patients with acute and chronic diabetic Charcot neuroarthropathy. Diabet Med 1997;14:527-531. [PubMed: 9223389]

14. Jirkovska A, Kasalicky P, Boucek P, Hosova J, Skibova J. Calcaneal ultrasonometry in patients with Charcot osteoarthropathy and its relationship with densitometry in the lumbar spine and femoral neck and with markers of bone turnover. Diabet Med 2001;18:495-500. [PubMed: 11472470]

15. Loder R. The influence of diabetes mellitus on the healing of closed fractures. Clin Orthop 1988:210216. [PubMed: 3289812](232)Review

16. Herskind AM, Christensen K, Norgaard-Andersen K, Andersen JF. Diabetes mellitus and healing of closed fractures. Diabete Metab 1992;18:63-64. [PubMed: 1563540]

17. Beam H, Parsons J, Lin S. The effects of blood glucose control upon fracture healing in the BB Wistar rat with diabetes mellitus. J Orthop Res 2002;20:1210-1216. [PubMed: 12472231]

18. Gandhi A, Beam HA, O'Connor JP, Parsons JR, Lin SS. The effects of local insulin delivery on diabetic fracture healing. Bone 2005;37:482-490. [PubMed: 16027060]

19. Gooch HL, Hale JE, Fujioka H, Balian G, Hurwitz SR. Alterations of cartilage and collagen expression during fracture healing in experimental diabetes. Connect Tissue Res 2000;41:81-91. [PubMed: 10992154] 
20. Herbsman H, Powers J, Hirschman A, Shaftan G. Retardation of fracture healing in experimental diabetes. J Surg Res 1968;8:424-431. [PubMed: 5673338]

21. Topping R, Bolander M, Balian G. Type X collagen in fracture callus and the effects of experimental diabetes. Clin Orthop 1994;308:220-228. [PubMed: 7955687]

22. Macey L, Kana S, Jingushi S, Terek R, Borretos J, Bolander M. Defects of early fracture-healing in experimental diabetes. J Bone Joint Surg Am 1989;71:722-733. [PubMed: 2659600]

23. Tyndall WA, Beam HA, Zarro C, O'Connor JP, Lin SS. Decreased platelet derived growth factor expression during fracture healing in diabetic animals. Clin Orthop Relat Res 2003:319-330. [PubMed: 12616077]

24. Lu H, Kraut D, Gerstenfeld L, Graves D. Diabetes interferes with the bone formation by affecting the expression of transcription factors that regulate osteoblast differentiation. Endocrinology 2003;144:346-352. [PubMed: 12488363]

25. Kayal RA, Tsatsas D, Bauer MA, et al. Diminished bone formation during diabetic fracture healing is related to the premature resorption of cartilage associated with increased osteoclast activity. $\mathrm{J}$ Bone Miner Res 2007;22:560-568. [PubMed: 17243865]

26. Graves DT, Naguib G, Lu H, Leone C, Hsue H, Krall E. Inflammation is more persistent in Type 1 diabetic mice. J Dent Res 2005;84:324-328. [PubMed: 15790737]

27. Gerstenfeld LC, Alkhiary YM, Krall EA, et al. Three-dimensional reconstruction of fracture callus morphogenesis. J Histochem Cytochem 2006;54:1215-1228. [PubMed: 16864894]

28. He H, Liu R, Desta T, Leone C, Gerstenfeld L, Graves D. Diabetes causes decreased osteoclastogenesis, reduced bone formation, and enhanced apoptosis of osteo-blastic cells in bacteria stimulated bone loss. Endocrinology 2004;145:447-452. [PubMed: 14525917]

29. Thrailkill KM, Liu L, Wahl EC, et al. Bone formation is impaired in a model of type 1 diabetes. Diabetes 2005;54:2875-2881. [PubMed: 16186388]

30. Kon T, Cho TJ, Aizawa T, et al. Expression of osteoprotegerin, receptor activator of NF-kappaB ligand (osteoprotegerin ligand) and related proinflammatory cytokines during fracture healing. J Bone Miner Res 2001;16:1004-1014. [PubMed: 11393777]

31. Barnes GL, Kostenuik PJ, Gerstenfeld LC, Einhorn TA. Growth factor regulation of fracture repair. J Bone Miner Res 1999;14:1805-1815. [PubMed: 10571679]

32. Maes C, Coenegrachts L, Stockmans I, et al. Placental growth factor mediates mesenchymal cell development, cartilage turnover, and bone remodeling during fracture repair. J Clin Invest 2006;116:1230-1242. [PubMed: 16614757]

33. Gerstenfeld LC, Cho TJ, Kon T, et al. Impaired fracture healing in the absence of TNF-alpha signaling: the role of TNF-alpha in endochondral cartilage resorption. J Bone Miner Res 2003;18:1584-1592. [PubMed: 12968667]

34. Apte SS. A disintegrin-like and metalloprotease (reprolysin type) with thrombos-pondin type 1 motifs: the ADAMTS family. Int J Biochem Cell Biol 2004;36:981-985. [PubMed: 15094112]

35. Kevorkian L, Young D, Darrah C, et al. Expression profiling of metalloproteinases and their inhibitors in cartilage. Arthritis Rheum 2004;50:131-141. [PubMed: 14730609]

36. Tortorella MD, Burn TC, Pratta MA, et al. Purification and cloning of aggrecanase-1: a member of the ADAMTS family of proteins. Science 1999;284:1664-1666. [PubMed: 10356395]

37. Lee ER. Proteolytic enzymes in skeletal development: histochemical methods adapted to the study of matrix lysis during the transformation of a "cartilage model" into bone. Front Biosci 2006;11:2538-2553. [PubMed: 16720332]

38. Li L, Khansari A, Shapira L, Graves D, Amar S. Contribution of interleukin-11 and protaglandin(s) in lipopolysaccharide-induced bone resorption in vivo. Infect Immun 2002;70:3915-3922. [PubMed: 12065535]

39. Lee FY, Choi YW, Behrens FF, DeFouw DO, Einhorn TA. Programmed removal of chondrocytes during endochondral fracture healing. J Orthop Res 1998;16:144-150. [PubMed: 9565087]

40. Follak N, Kloting I, Wolf E, Merk H. Improving metabolic control reverses the histomorphometric and biomechanical abnormalities of an experimentally induced bone defect in spontaneously diabetic rats. Calcif Tissue Int 2004;74:551-560. [PubMed: 15354863]

41. Irwin R, Lin HV, Motyl KJ, McCabe LR. Normal bone density obtained in the absence of insulin receptor expression in bone. Endocrinology 2006;147:5760-5767. [PubMed: 16973725] 
42. Follak N, Kloting I, Wolf E, Merk H. Histomorphometric evaluation of the influence of the diabetic metabolic state on bone defect healing depending on the defect size in spontaneously diabetic BB/ OK rats. Bone 2004;35:144-152. [PubMed: 15207750]

43. Dixit P, Ekstrom R. Retardation of bone fracture healing in experimental diabetes. Indian J Med Res 1987;85:426-435. [PubMed: 3623654] 


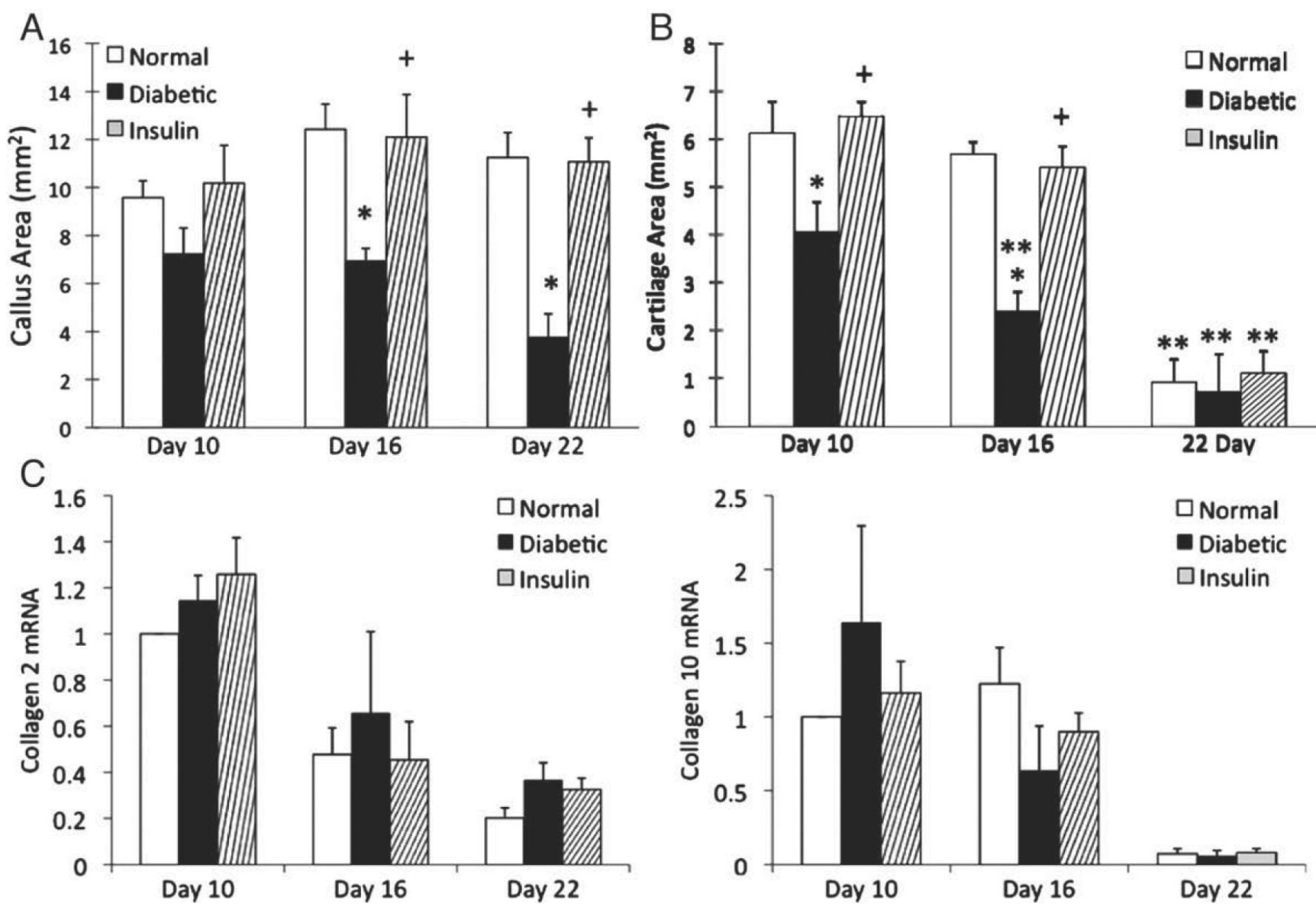

Fig. 1.

(A) Comparison of callus size in diabetic, normoglycemic and diabetic insulin treated mice with femoral fracture. Callus area was measured in H\&E stained cross-sections obtained from 3 points sampled at the fracture line and $0.5 \mathrm{mmproximal}$ and distal and presented as the sum of these three sites. (B) Comparison of cartilage area in diabetic, normoglycemic and diabetic insulin treated mice with femoral fracture. The area of cartilage within each callus was measured in safranin-O/fast green stained sections in the same manner. The individual measurements were averaged to establish a value of total callus area per animal for each of the 3 time points examined. Data are expressed as mean \pm SEM. * indicates a significant difference between normal and diabetic $(P<0.05) .+$ indicates significant difference between insulin treated and untreated diabetic animals $(P<0.05)$. ** indicates a significant difference compared with the previous time point within a group $(P<0.05)$. (C)mRNA levels of markers of cartilage formation. mRNA levels were measured by real-time PCR for Collagen 2 and collagen 10 . Each marker was evaluated in 3 separate experiments using 3 mice in each set (total $n=3$ ) and the results are expressed as mean \pm SEM. 

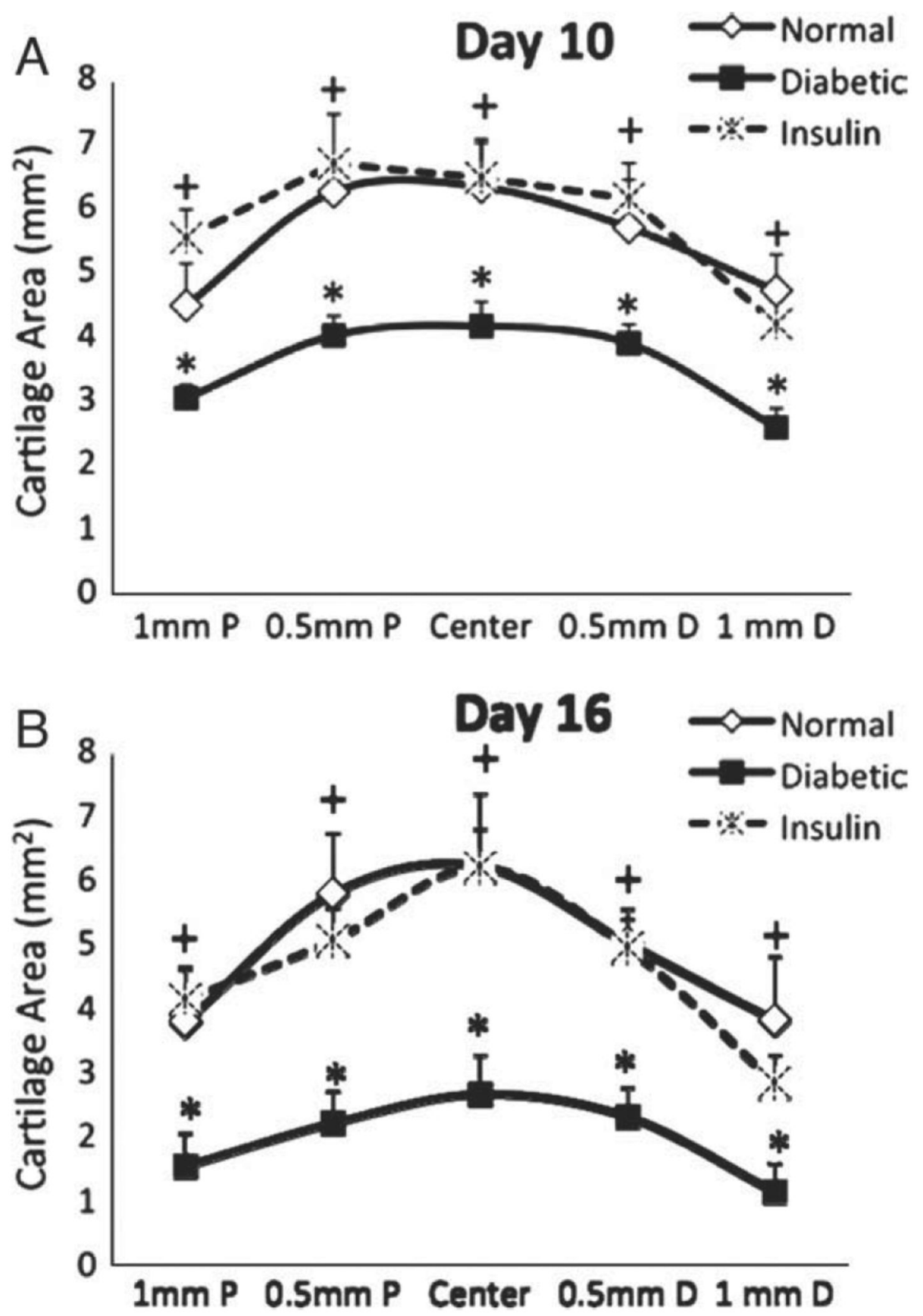

Fig. 2.

Comparison of cartilage area at $0.5 \mathrm{~mm}$ intervals in diabetic, normoglycemic and diabetic insulin treated mice with femoral fracture. Cartilage area was measured at 5 points sampled at the fracture line and $0.5 \mathrm{~mm}$ and $1.0 \mathrm{~mm}$ proximal and distal at the indicated time point. Data are expressed as mean \pm SEM. * indicates a significant difference between normal and diabetic $(P<0.05) .+$ indicates a significant difference between insulin treated and untreated diabetic animals $(P<0.05)$. 

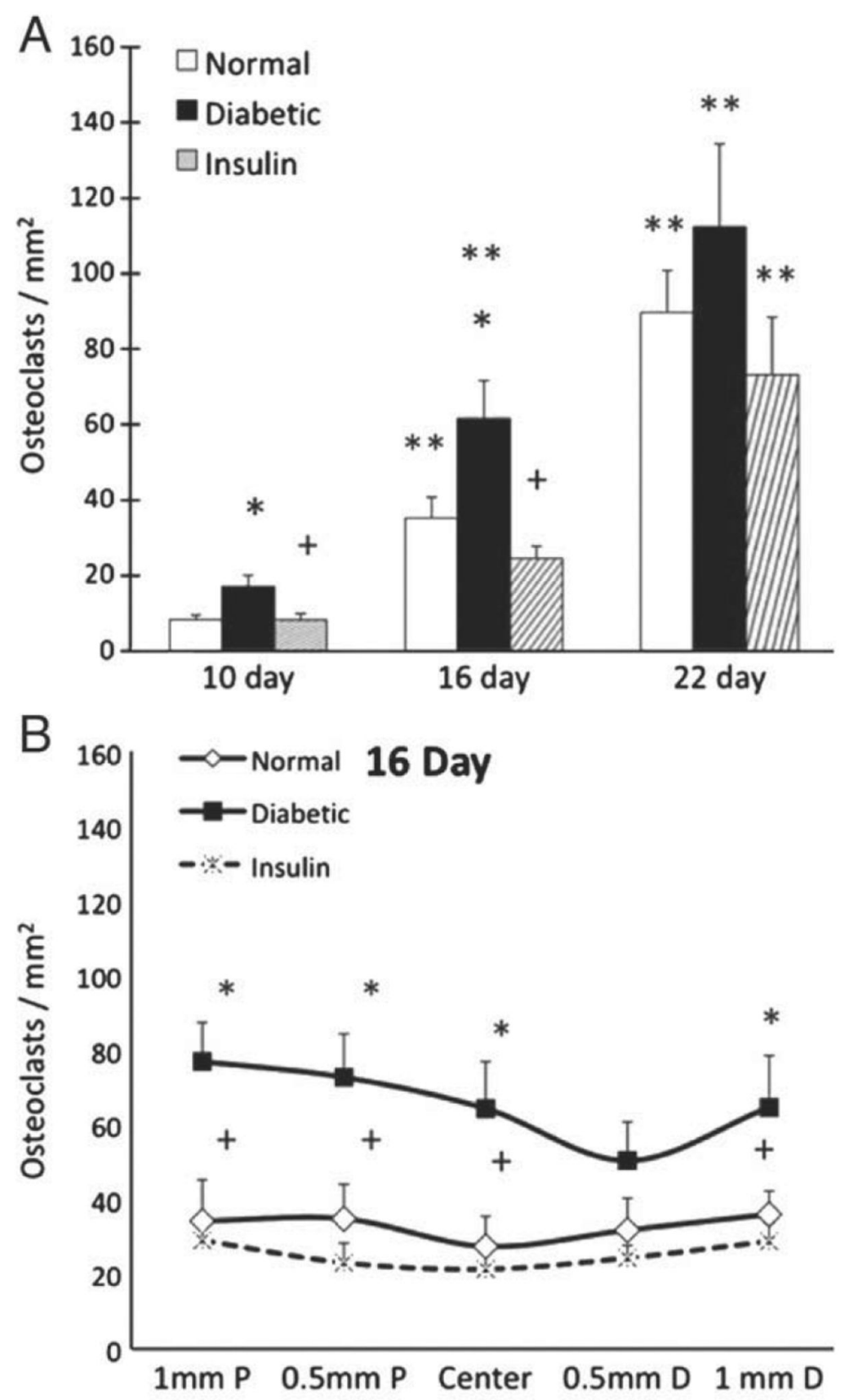

Fig. 3.

Comparison of osteoclast numbers in diabetic, normoglycemic and diabetic insulin treated mice. (A) The number of osteoclasts was measured in TRAP stained sections and normalized to cartilage and new bone area. For a given animal three points along a $1 \mathrm{~mm}$ length of the callus were sampled, the individual counts were averaged to establish a value of total osteoclast number per animal for each of the 3 time points. (B) Detailed comparison of osteoclast numbers at $0.5 \mathrm{~mm}$ intervals in diabetic, normoglycemic and diabetic insulin treated mice with femoral fracture on day 16. Data are expressed as mean \pm SEM. * indicates a significant difference between normal and diabetic $(P<0.05)$. + indicates a significant difference between insulin 
treated and untreated diabetic animals $(P<0.05)$.** indicates a significant difference compared with the previous time point within a group $(P<0.05)$. 

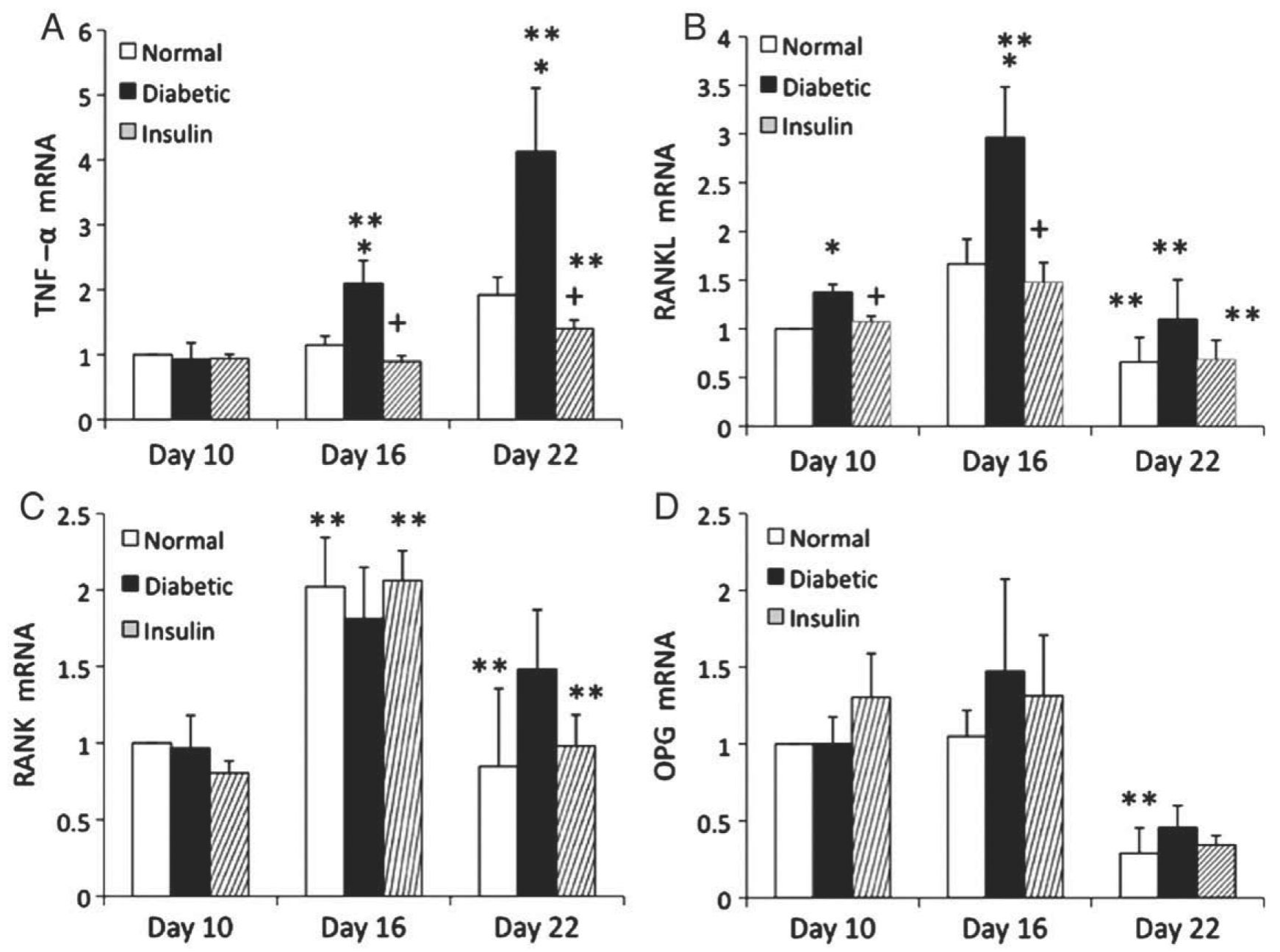

Fig. 4.

mRNA levels of cytokines that regulate osteoclastogenesis in femoral fracture. mRNA levels were measured by real-time PCR for TNF- $\alpha$, RANKL, RANK and OPG. Each cytokine was evaluated in 3 separate experiments using 3 mice in each set (total $n=3$ ) and the results are expressed as mean \pm SEM. * indicates a significant difference between normal and diabetic $(P<0.05) .+$ indicates a significant difference between insulin treated and untreated diabetic animals $(P<0.05)$. ** indicates a significant difference compared with the previous time point within a group $(P<0.05)$. 

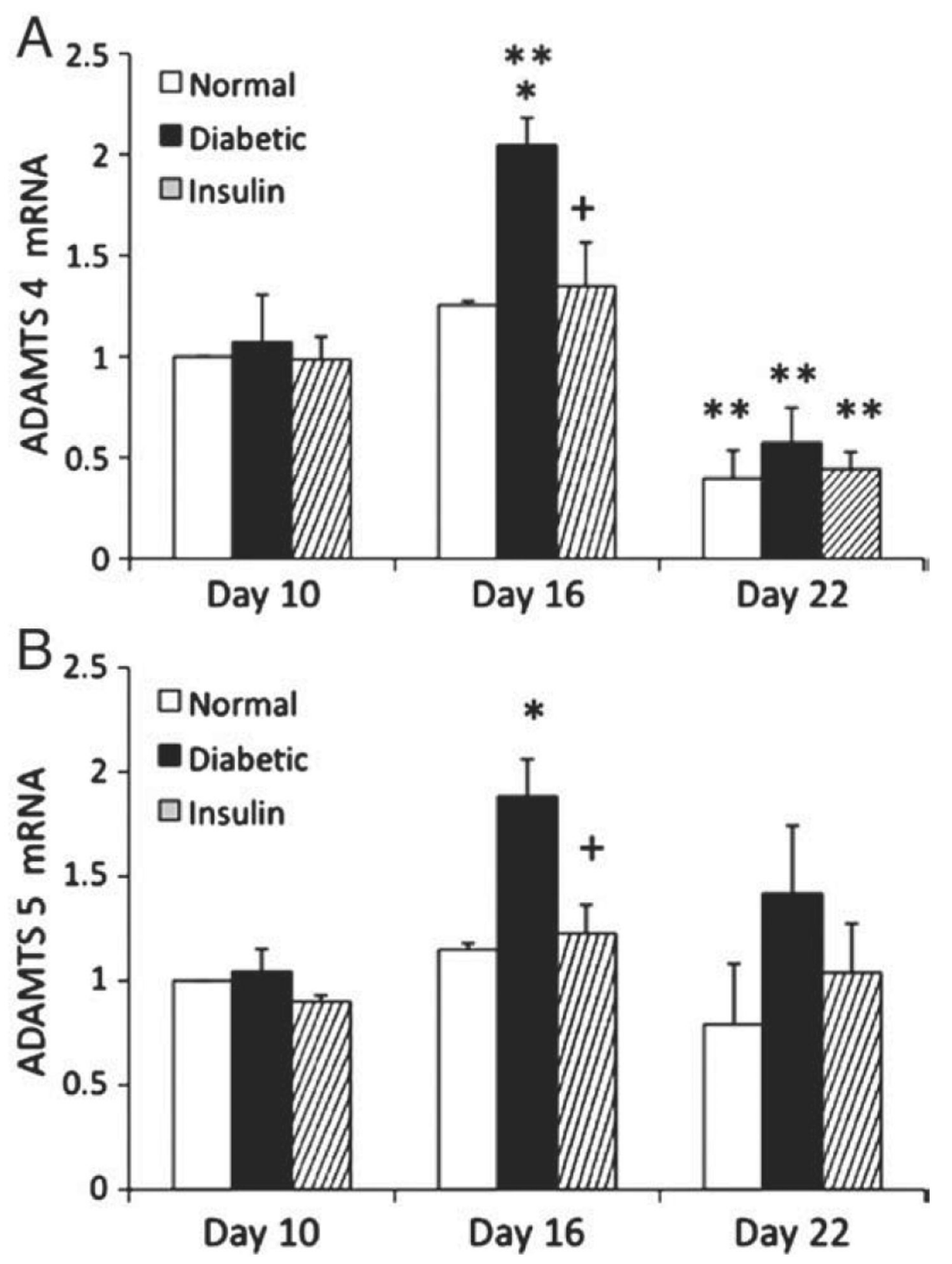

Fig. 5.

mRNA levels of enzymes associated with cartilage degradation during fracture repair. mRNA levels were measured by real-time PCR for ADAMTS-4 and ADAMTS-5. Each enzyme was evaluated in 3 separate experiments using 3 mice each $(n=3)$ and the results are expressed as mean \pm SEM. *indicates a significant difference between normal and diabetic $(P<0.05) .+$ indicates a significant difference between insulin treated and untreated diabetic animals $(P<0.05)$. ** indicates a significant difference compared with the previous time point within a group $(P<0.05)$. 

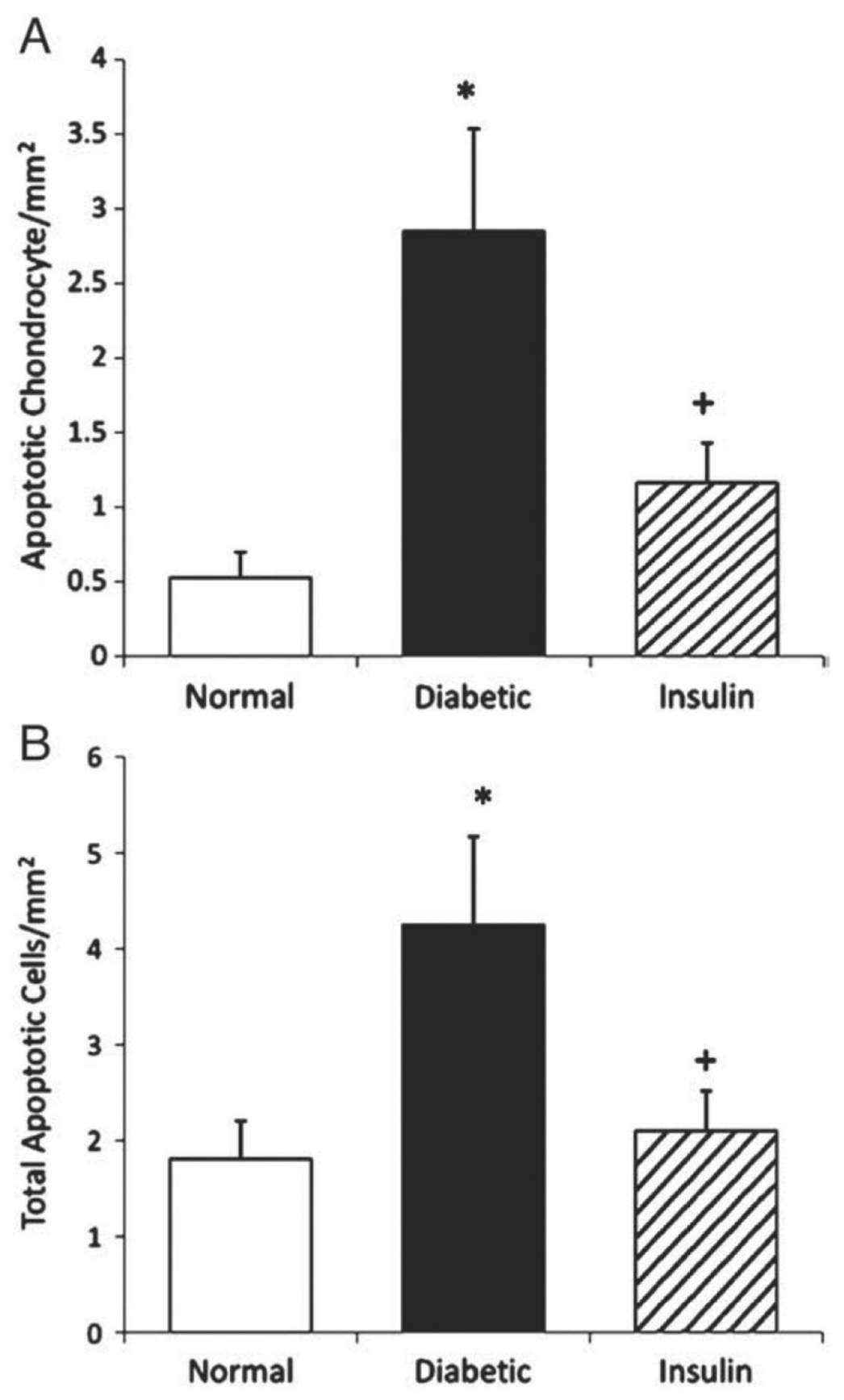

Fig. 6.

Comparison of apoptotic cell numbers in diabetic, normoglycemic and diabetic insulin treated mice with femoral fracture. The number of apoptotic cells within each callus was measured in TUNEL stained sections combined with safranin-O/fast green stain to distinguish chondrocytes on day 16 specimens. For a given animal a section from the midline of the callus was chosen for the assay. (A) Apoptotic chondrocytes were counted and normalized to cartilage area. (B) The total number of apoptotic cells was normalized to callus area. Data are expressed as mean \pm SEM. * indicates a significant difference between normal and diabetic $(P<0.05)$. + indicates a significant difference between insulin treated an untreated diabetic animals $(P<0.05)$. 


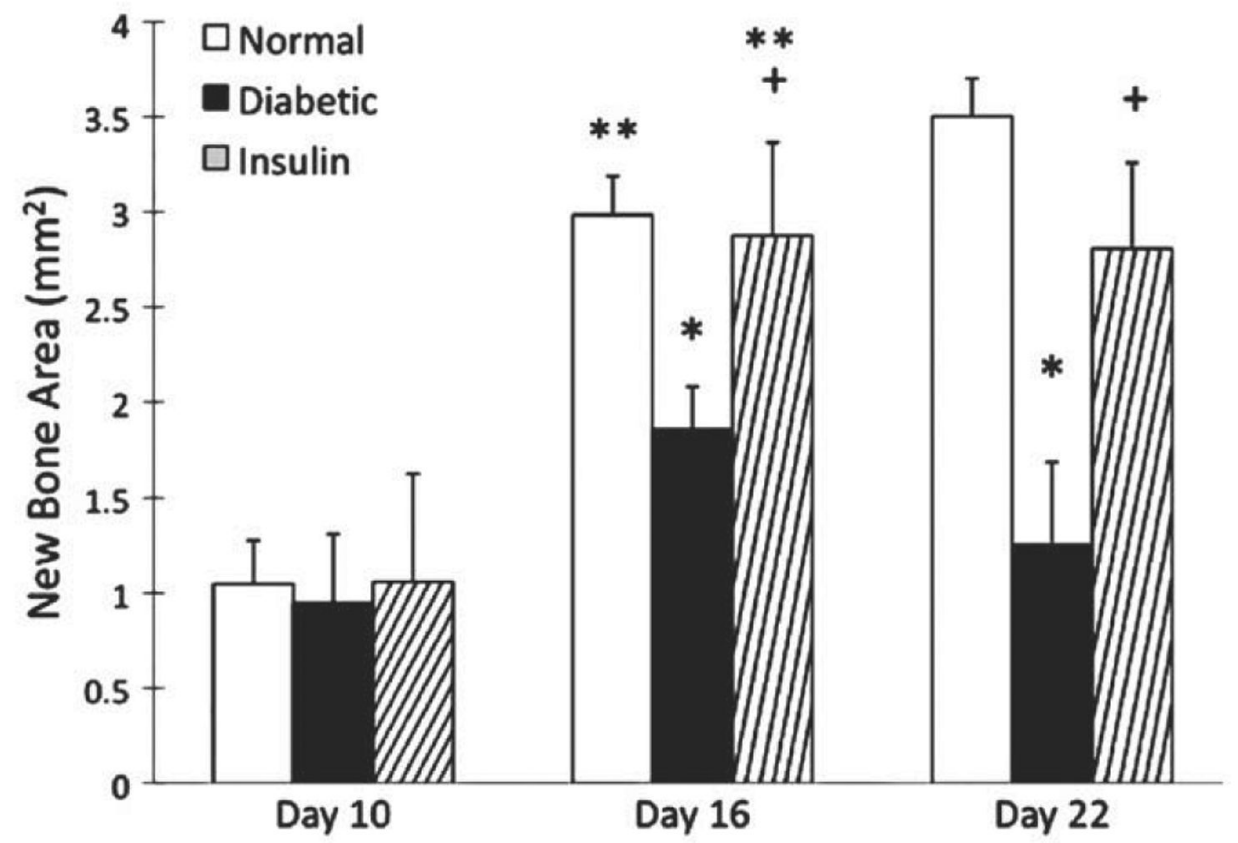

Fig. 7.

Comparison of new bone area in diabetic, normoglycemic and diabetic insulin treated mice with femoral fracture. The area of new bone within each callus was measured in Masson's trichrome stained sections in the same manner as described in Fig. 1. Data are expressed as mean \pm SEM. * indicates a significant difference between normal and diabetic $(P<0.05) .+$ indicates a significant difference between insulin treated and untreated diabetic animals $(P<0.05)$. ** indicates a significant difference compared with the previous time point within a group $(P<0.05)$. 
Table 1

Serum glucose and glycosylated hemoglobin values

\begin{tabular}{llll}
\hline & Normoglycemic & Diabetic & Diabetic + insulin \\
\hline Serum glucose & $147 \pm 3.2$ & $490 \pm 12.9$ & $108 \pm 6.4$ \\
Glycosylated hemoglobin & $6.2 \pm 0.18$ & $12.2 \pm 0.24$ & $7.4 \pm 0.25$ \\
\hline
\end{tabular}

Serum glucose levels were measured weekly starting two weeks after induction of diabetes and the mean values are given for each group. Glycosylated hemoglobin levels were measured at the time of euthanasia from blood obtained by cardiac puncture. 